$\mathrm{DE} 86 \quad 002011$

\title{
Very Low Frequency Magnetotelluric and Dipole-Dipole Responses of Three-Dimensional Thin-Layer Resistivity Structure Modeled Using Finite Elements
}

Philip E. Wannamaker

\author{
Earth Science Laboratory \\ University of Utah Research Institute \\ 391 Chipeta Way, Suite C \\ Salt Lake City, Utah 84108
}

\section{DISCLAIMER}

This report was prepared as an account of work sponsored by an agency of the United States Government. Neither the United States Government nor any agency thereof, nor any of their employees, makes any warranty, express or implied, or assumes any legal liability or responsibility for the accuracy, completeness, or usefulness of any information, apparatus, product, or process disclosed, or represents that its use would not infringe privately owned rights. Reference herein to any specific commercial product, process, or service by trade name, trademark, manufacturer, or otherwise does not necessarily constitute or imply its endorsement, recommendation, or favoring by the United States Government or any agency thereof. The views and opinions of authors expressed herein do not. necessarily state or reflect those of the United States Government or any agency thereof.

September, 1985 


\section{DISCLAIMER}

This report was prepared as an account of work sponsored by an agency of the United States Government. Neither the United States Government nor any agency Thereof, nor any of their employees, makes any warranty, express or implied, or assumes any legal liability or responsibility for the accuracy, completeness, or usefulness of any information, apparatus, product, or process disclosed, or represents that its use would not infringe privately owned rights. Reference herein to any specific commercial product, process, or service by trade name, trademark, manufacturer, or otherwise does not necessarily constitute or imply its endorsement, recommendation, or favoring by the United States Government or any agency thereof. The views and opinions of authors expressed herein do not necessarily state or reflect those of the United States Government or any agency thereof. 


\section{DISCLAIMER}

Portions of this document may be illegible in electronic image products. Images are produced from the best available original document. 
This report was prepared to document work sponsored by the United States Government. Neither the United States nor its agent, the United States Department of Energy, nor any Federal employees, nor any of their contractors, subcontractors or their employees, makes any warranty, express or implied, or assumes any legal liability or responsibility for the accuracy, completeness, or usefulness of any information, apparatus, product or process disclosed, or represents that its use would not infringe privately owned rights.

\section{NOTICE}

Reference to a company or product name does not imply approval or recommendation of the product by the University of Utah Research Institute or the U.S. Department of Energy to the exclusion of others that may be suitable. 


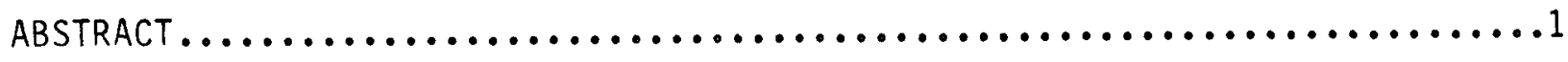

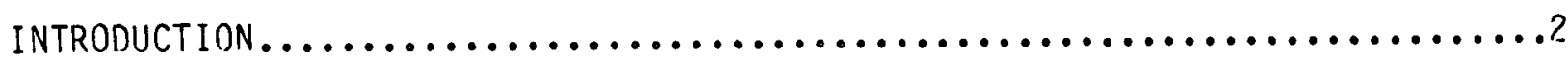

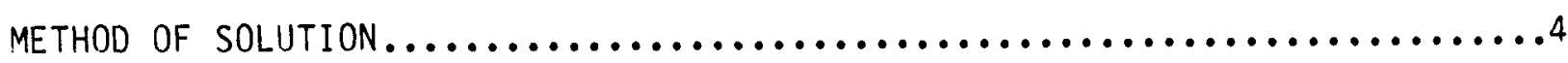

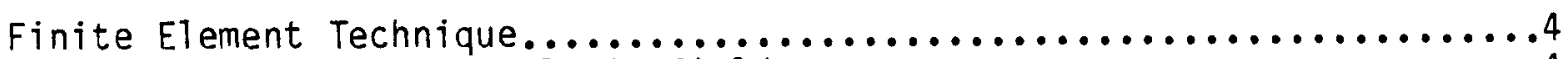

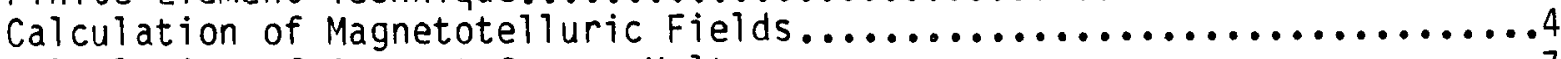

Calculation of Current Source Voltages..........................

MAGNETOTELLURIC RESPONSES................................

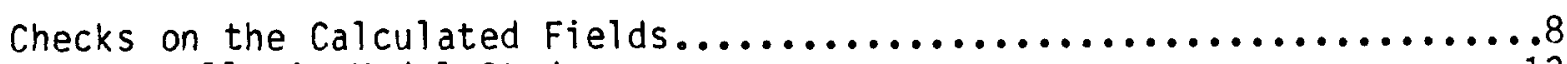

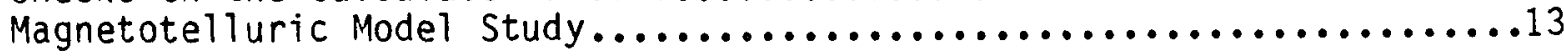

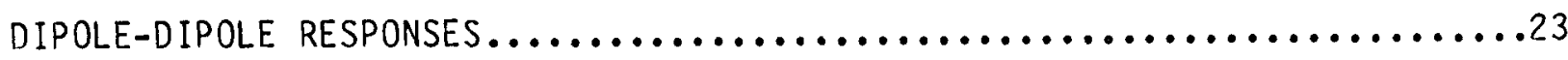

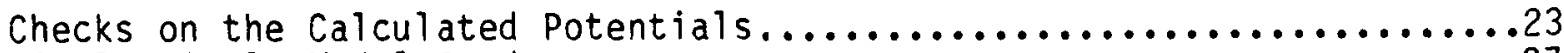

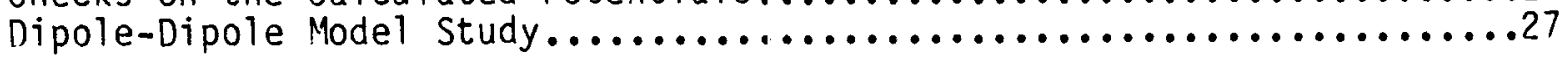

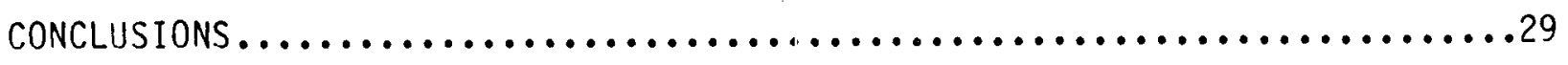

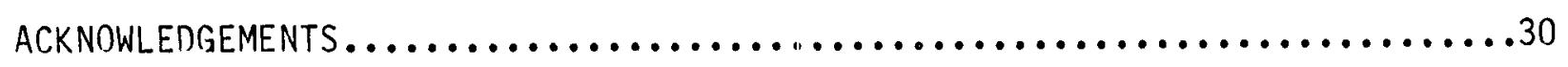

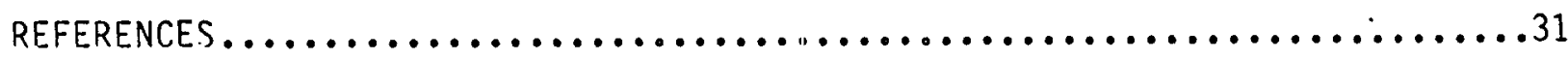




\section{ABSTRACT}

An algorithm to simulate the very low frequency magnetotelluric and dipole-dipole responses of three-dimensional thin-layer inhomogeneity has been developed from an existing finite element program used to simulate resistivity/IP signatures of 2-D earth sections. The 3-D body is confined to and extends uniformly across a thin layer overlain by air and underlain by infinite resistivity. Electric fields are obtained through differentiation of piecewise parabolas fit to nodal voltages while magnetic fields arise from integration of the free-space Green's tensor over secondary current perturbations throughout the thin layer. Dipole-dipole apparent resistivities arise through application to nodal voltages of the logarithmic geometric factors appropriate to continuously grounded line sources of current. Checks on accuracy of the simulation utilize analytic solutions, 2-D AC plane-wave results using finite elements, and a surface integral equations technique.

Low-frequency limits to 3-D thin-layer responses are useful to know because they often represent maxima in anomaly strength or areal extent. It is hazardous however to apply such a thin-layer program beyond simple orderof-magnitude forward calculations. For example, supposition that 2-D TM mode interpretation approaches to defining deep structure below surficial heterogeneity may suffer pitfalls due to the 3-D nature of the surficial structure cannot be upheld just on the basis of very low frequency, 3-D thin-layer calculations. This is because any single-frequency profile of apparent resistivities chosen as TM across a 3-D structure may be fit precisely by an equivalent 2-D geometry whose entire resistivity variation is limited to the same class of outcropping thin-layer inhomogeneity. All that may be concluded firmly by these thin-layer studies is that errors in estimates of the 3-D surficial structure could result from a 2-D TM application. 


\section{INTRODUCTION}

Thin-layer algorithms have been developed by many geophysicists in order to model inhomogeneous resistivity structure in nature corresponding to oceancontinent margins, sedimentary distributions, or other structure which is essentially outcropping and whose thickness is much less than its areal extent (Weidelt, 1971; Vasseur and Weidelt, 1977; Dawson and Weaver, 1979; Ranganayaki and Madden, 1980; Hermance, 1982; Park et a1., 1983; Mckirdy et al., 1985). An example of these algorithms is that described by Hermance (1982) which considers only the zero-frequency limit of the EM response. Furthermore, this program assumes the thin-layer structure overlays a substratum of infinite resistivity and thus disallows vertical flow of anomalous current.

Due to its ease of construction, we have developed such a $3-0$ thin-layer program from an existing finite element code for solving the 2-D DC resistivity problem (Rijo, 1977; Fox et al., 1980). Both electric and magnetic anomalies are computed as well as responses of 3-D thin-layer structure to dipole-dipole electrode arrays. A simple example of the 3-D thin-layer inhomogeneity which our program can simulate appears in Figure 1. This report describes our algorithm, examines accuracy of its electric and magnetic field and of its dipole potential calculations, and points out peculiarities and limitations in applying this class of model structure to field electrical observations. 
0

0

0

OUTCROP $\rho_{1}=100 \Omega \cdot m$

ALLUVIUM $\rho_{2}=5 \Omega \cdot m$

0

0

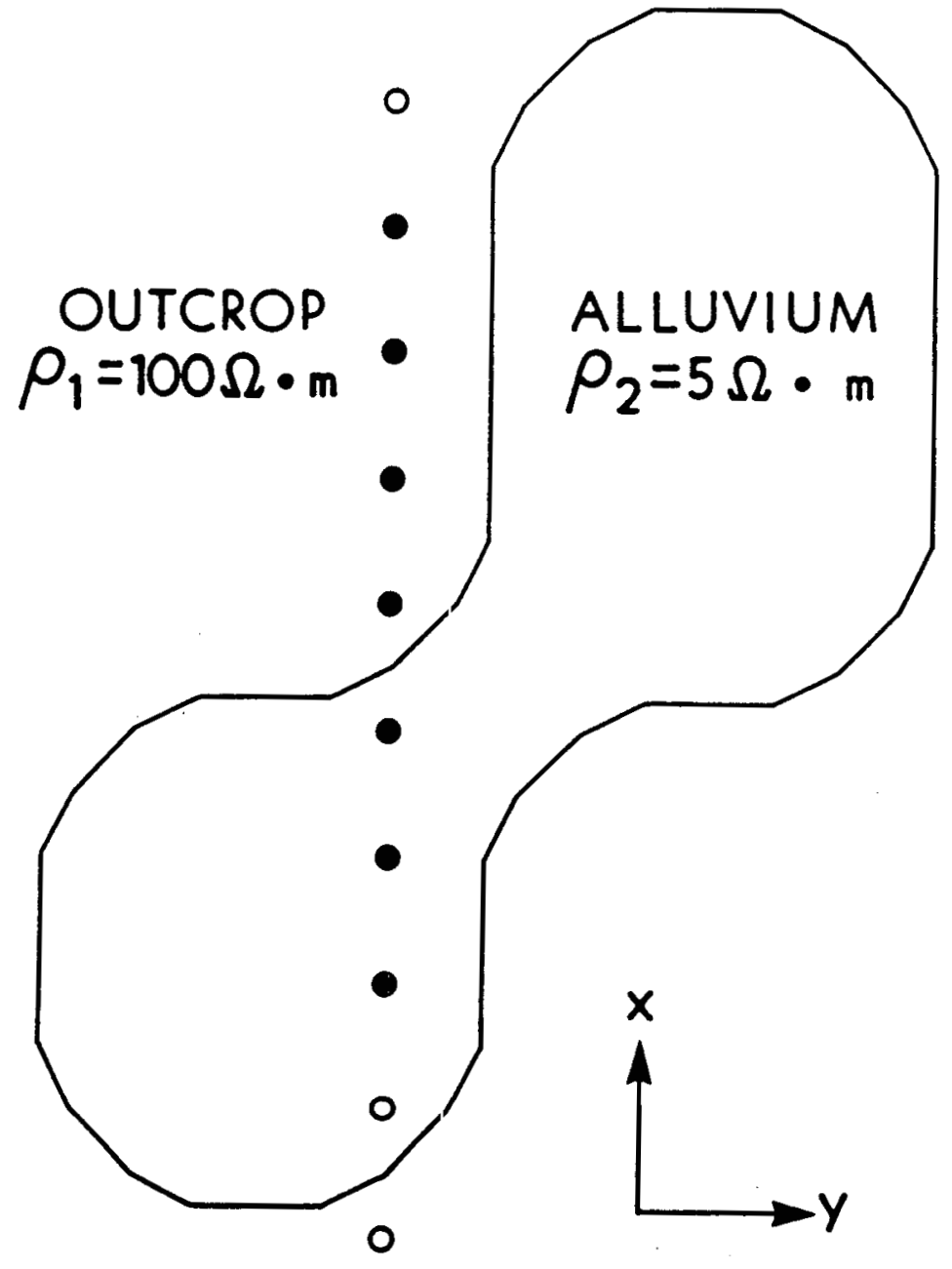

0

o

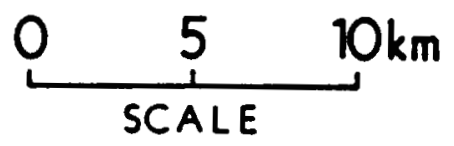

0

Figure 1. Example of 3-D thin-layer structure as modeled using our finite element program. The structure varies only in plan view, is of uniform thickness, and is overlain by air and underlain by a basement of infinite resistivity. Possible electrode locations for dipole-dipole modeling are shown also. 
METHOD OF SOLUTION

Finite Element Technique

Our 3-D thin-sheet program makes use of the finite element method to derive a system of linear equations which approximates the governing Poisson's partial differential equation for total electrostatic potential. This method in our case employs linear interpolation of the unknown potential over triangular subdomains in conjunction with the Galerkin weighting technique and the process of static condensation to provide the system of equations (Rijo, 1977; Huebner and Thornton, 1982). The solution of this linear system gives the approximate potentials at the nodes of the discretized domain. Under the assumption of no vertical current flow or field variation across the thin sheet, the 3-D problem reduces to a 2-D solution over the area of the sheet (cf. Hermance, 1982). Field quantities are obtained subsequently through voltage differentiation (electric) and voltage integration (magnetic). Dipole-dipole apparent resistivities result from bipole voltage differences multiplied by the appropriate array geonetric factors.

\section{Calculation of Magnetotelluric Fields}

To provide electric field variations for the magnetotelluric problem, total voltage distributions are computed at the outset with boundary conditions corresponding to a uniform inciderit voltage gradient first in the $-y$ and then in the $-x$ directions. The strength of the gradient is the electric field at the surface of the layered host for the body (including the infinite resistivity underlying the body and any deeper layers) at the nominal frequency (10w) of simulation. To be accurate, the boundary voltage gradient across any thin-layer structure which extends to the borders of the mesh should be multiplied by the ratio of the thin-layer resistivity to the 
resistivity $p_{1}$ of the uppermost layer (Figure 1 ). This preserves continuity of total normal current density.

From the total voltages resulting from solution of the system of finite element equations, $x$ - and $y$-components of electric field are calculated by fitting parabolas to this nodal potential plus those immediately to the sides, and then differentiating these functions. The accuracy of this method has been demonstrated for computing auxiliary fields in the 2-D AC plane-wave problem (Wannamaker et al., 1985) and by checks presented in this report. However, to simplify the coding and to avoid electric field discontinuities, our thin-layer program does not compute fields at nodes on resistivity boundaries.

Magnetic fields are obtained through integration of an appropriate Green's tensor over the current density. Two approaches are possible. One is that of Wannamaker et a1. (1984a) in which the Green's tensor for the layered earth (here the thin-layer background $p_{1}$ over the infinitely resistive substratum and any deeper layers) is integrated over just the product of the total electric field and the conductivity difference between the anomalous thin layer structure (of resistivity $\rho_{?}$ in Figure 1) and the thin-layer background medium. In the second approach, the Green's tensor for free-space is integrated over the electric current density throughout the entire domain of simulation (the earth). In the algorithm described herein, the latter method is utilized. Since the basal half-space is of infinite resistivity and frequency is essentially zero, there are no anomalous current perturbations in it or below that matter for the secondary magnetic fields. Thus, electric current density need only be integrated over the thin layer. The Green's tensor elements of interest are the low-frequency asymptotes of equations (A- 
27a-h) of Wannamaker et al. (1984a). These are simple analytic functions so that time-consuming Hankel transformation is avoided.

To enable an accurate integration, a formulation in terms of secondary current perturbations was implemented. The magnetic field $\vec{H}_{L}$ produced by the total current throughout the thin layer is expressed as

$$
\vec{H}_{L}=\left\{\tilde{G}^{H} \cdot \overrightarrow{E E} d v^{\prime}\right.
$$

where $\widetilde{G}^{H}$ is the free-space Green's tensor, $\sigma$ is total electrical conductivity and $\vec{E}$ is the total vector electric field in the thin layer. Both conductivity and electric field can be split into background and anomalous portions so that (1) may be written

$$
\vec{H}_{L}=\left(\tilde{G}^{H} \cdot\left[\sigma_{1} \vec{E}_{i}+\sigma_{1} \vec{E}_{s}+\left(\sigma-\sigma_{1}\right) \vec{E}_{i}+\left(\sigma-\sigma_{1}\right) \vec{E}_{s}\right] d v^{\prime},\right.
$$

in which $\sigma_{1}$ is the background conductivity (inverse of resistivity $\rho_{1}$ ) and in which $\vec{E}_{j}$ and $\vec{E}_{S}$ are the incident and secondary vector electric fields in the thin layer.

The first term in the integral is just the contribution to the incident magnetic field $\vec{H}_{i}$ by the current density in the thin layer in the absence of any inhomogeneity. Since $\vec{H}_{j}$ is computed readily using the recursive relations of Ward (1967), it is omitted from the numerical integration of our algorithm. It is the remaining three terms in equation (2) which have been integrated to give the anomalous magnetic fields. To perform the integration, the midpoint rule has been employed. Sixteen midpoints per rectangular element are selected with the exception of the four elements immediately surrounding the field point, where 100 midpoints are used. Finally, to improve accuracy further, $\mathrm{H}$-fields are computed at a height of one thin-layer thickness over 
the thin-layer structure.

Calculation of Current Source Voltages

Under the assumption of no vertical variation of potential across the thin layer, the current sources in our 3-D algorithm are distributed uniformly across the thin layer. In other words, the current source is a uniform line grounded along its entire length from top to bottom of the layer. This simplification avoids repeated calculation of mesh potentials as a function of spatial wavenumber along the strike direction, with subsequent inverse Fourier transformation of the potentials, which is the traditional method of DC resistivity simulation for point current sources over a 2-D earth (Rijo, 1977). Given the highly resistive basement, the departure between point and line current sources should be small if source and receiver electrodes are several thin-layer thicknesses from any lateral resistivity boundaries.

The potential about a uniformly grounded line source across a thin layer with no resistivity inhomogeneity decays logarithmically with distance from the source (Kellogg, 1953). A pole-bipole apparent resistivity, considered in an upcoming section on verification of potential calculations by our algorithm, is defined as follows:

$$
\rho_{a}=\frac{2 \pi}{I} \Delta V / \ln \left(\frac{r_{2}}{r_{1}}\right)
$$

where $I$ is the current strength per unit length, $\Delta V$ is the voltage difference across the ends of the bipole receiver, and $r_{2}$ and $r_{1}$ are radial distances from the current source to the ends of the bipole. Equation (3) is readily generalized to give $\rho_{a}$ for the dipole-dipole array. The expression is

$$
\rho_{a}=\frac{2 \pi}{I} \Delta V / \ln \left(\frac{(n+1)^{2}}{n(n+2)}\right)
$$

where $n$ is the $n$-spacing of the current and voltage electrode pairs. 


\section{MAGNETOTELLURIC RESPONSES}

Checks on the Calculated Fields

To verify the ability of the algorithm to compute accurate electric and magnetic fields over resistivity inhomogeneity, I consider first a finite element approximation to a conductive circular disk (Figure 2). For this check, the resistivity $\rho_{1}$ of the host is $100 \Omega-m$ while that of the conductive disk is $1 . \Omega-m$. Receiver points are marked by $x^{\prime} s$. Due to the assumption of air above and infinite resistivity below the thin layer, the electric field portion of this finite element simulation may be compared to the analytic response of a cylinder in a whole space, the solution to which is given by Ward (1967). The analytic and finite element calculations appear in Figures 3 and 4 for both $x$ and $y$ polarizations of incident electric field. The agreement between the two calculations is excellent indicating that electric field variations for 3-0 thin-layer structure are well-simulated by the algorithm.

To check the accuracy of the thin-layer program for computing magnetic fields, the response over a 2-D conductive strip was calculated and compared to that above a similar strip overlying a basement of $10^{20} \Omega-m$ resistivity as simulated by our 2-D AC plane-wave finite element program (Wannamaker et al., 1985 ). The nominal frequency of simulation by the AC plane-wave program was $0.01 \mathrm{~Hz}$, but the results have been normalized by the magnitude of the incident electric field at the surface. The comparison between the two sets of calculations again is very good to excellent (Figure 5), with the greatest disagreement being in $\mathrm{H}_{2}$ directly over the edge of the strip (integration allows H-field calculation over resistivity boundaries). The integral equation calculation in fact may be the more accurate since some aliasing of very extreme $H$-field variations will occur in the $A C$ plane-wave program due to the 


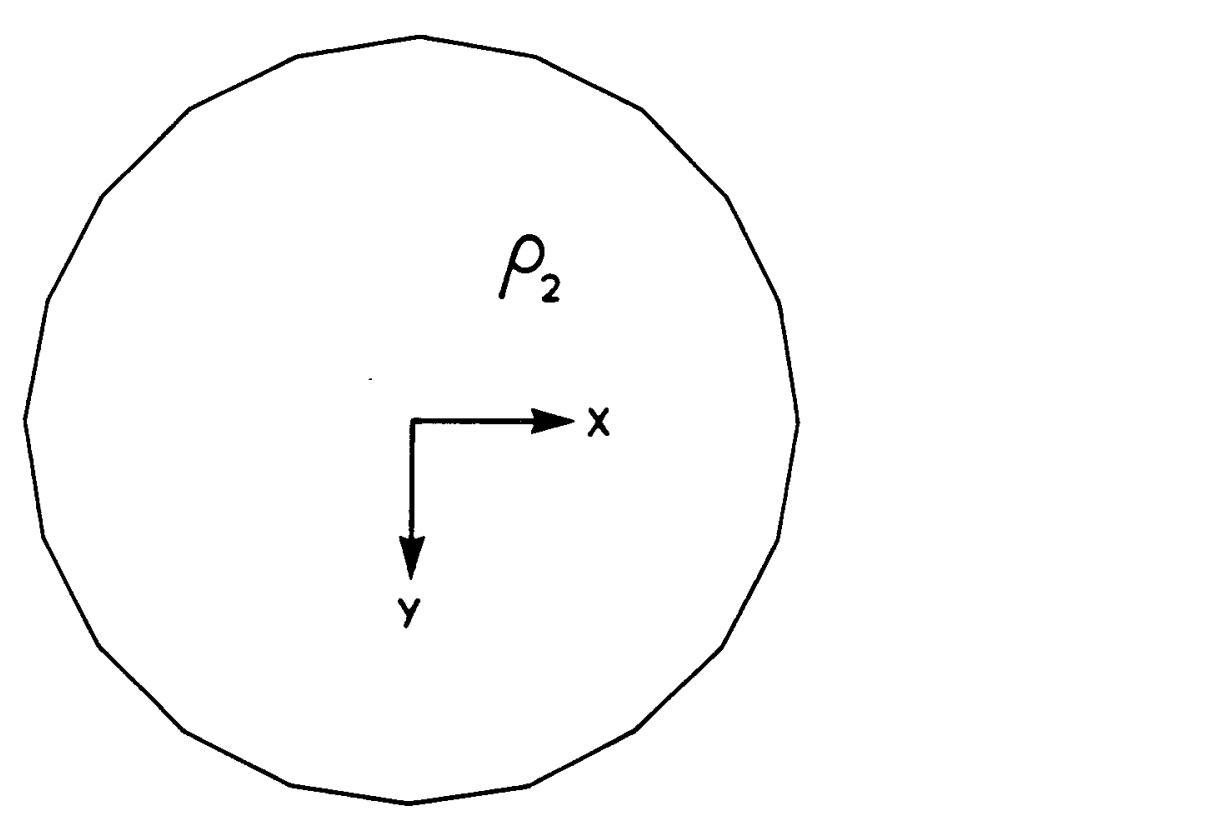

$\rho_{1}$

$\underset{1}{0} \quad 2 \mathrm{~km}$

Figure 2. Finite element approximation of conducting circular disk for comparison with analytic solution for electric fields of a cylinder. Locations of field points of finite element solution are shown with $x$ 's. Mesh extends approximately $50 \mathrm{~km}$ in the $\pm x$ and \pm $y$ directions for application of uniform field boundary conditions. The thickness $T$ of the structure is $0.1 \mathrm{~km}$. 


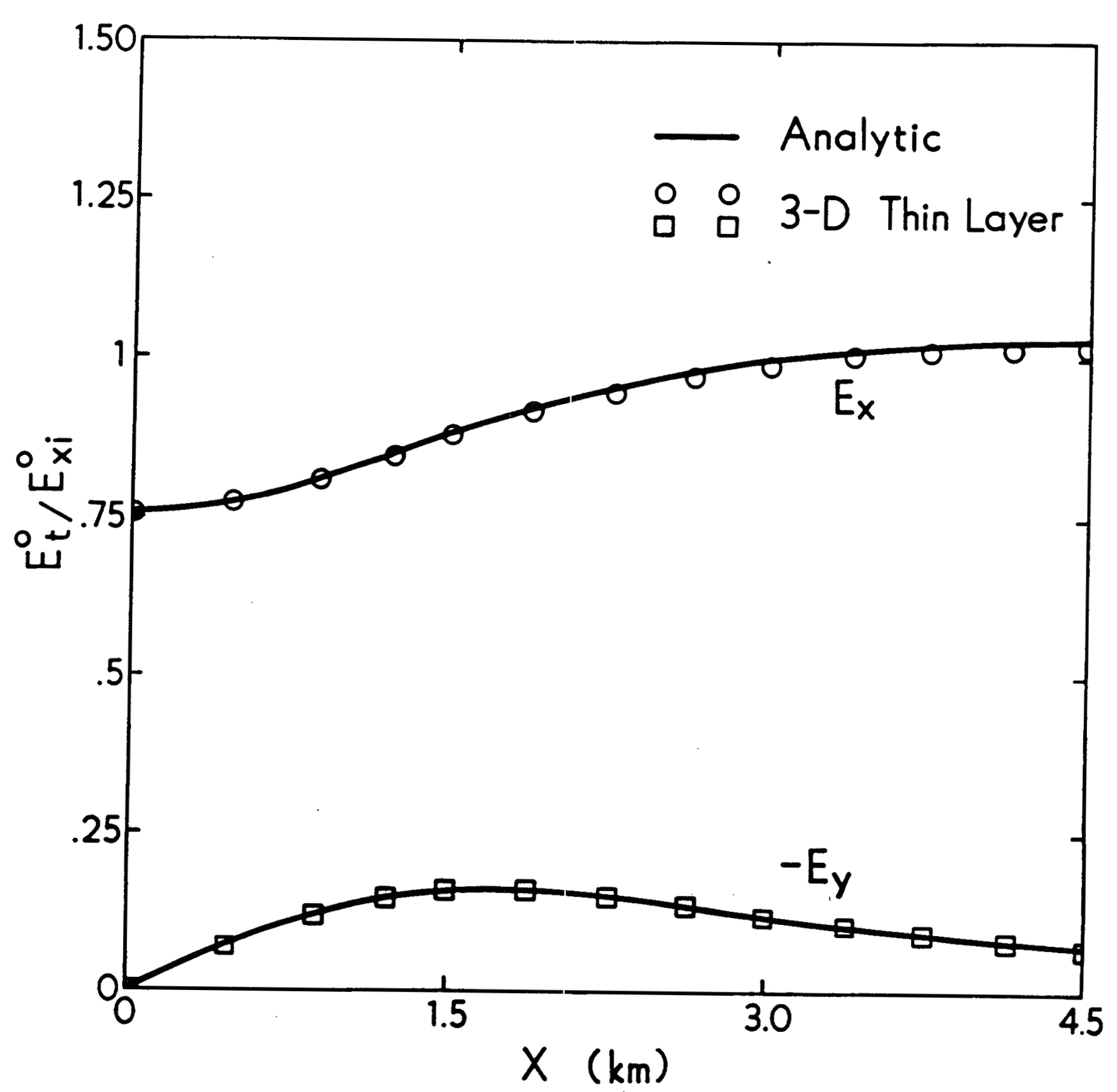

Figure 3. Total electric field response of conductive disk at field points shown in Figure 2 normalized by the incident electric field along the positive $x$-axis. Label $-E_{y}$ indicates field is directed along negative $y$-axis. Solid curves are analytic responses of cylinder. 


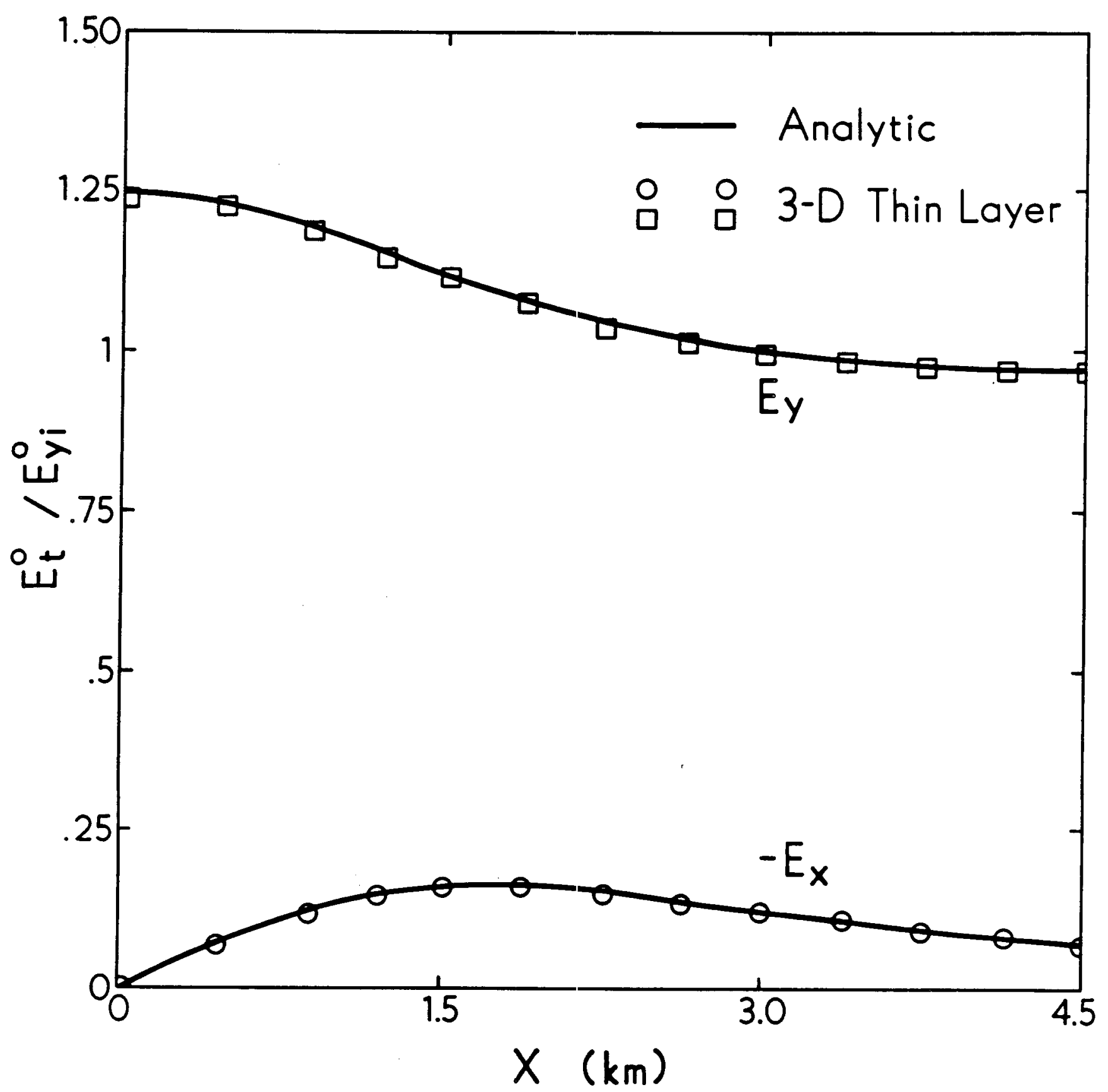

Figure 4. Total electric field response of conductive disk normalized by the incident electric field along the positive $y$-axis. Label $-E_{x}$ indicates field is directed along negative $x$-axis. Solid curves are analytic responses of cylinder. 

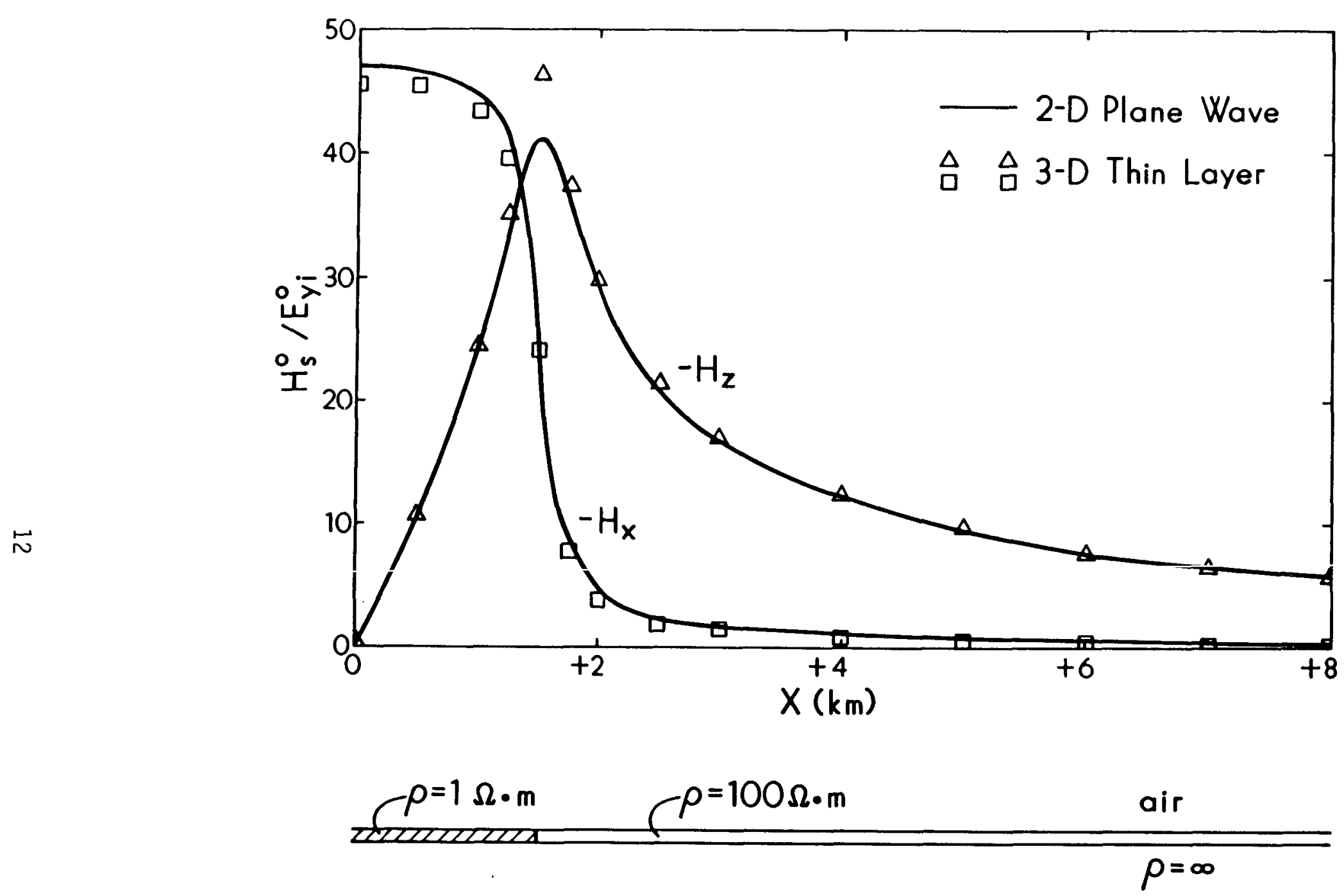

Figure 5. Secondary magnetic fields above 2-D conductive strip computed through integration over thin-sheet current perturbations compared with calculations using 2-D AC plane-wave results. Thin-layer structure, whose cross-section is shown at bottom, was discretized by elements $0.25 \mathrm{~km}$ wide over the region of inhomogeneity and fieldpoints, but with elements increasing beyond that to approximately $50 \mathrm{~km}$ in the $\pm x$ and $\pm y$ directions for application of uniform field boundary conditions. In the 2-D plane-wave program, the thinlayer was discretized vertically into five rows of elements. Labels $-H_{x}$ and $-H_{z}$ indicate secondary fields are directed along negative coordinate axes. The thickness $T$ of the st fucture is $0.1 \mathrm{~km}$ and the field values are normalized by the magnitude of the incident electric field $E_{y i}^{0}$. 
inumerical differencing of electric fields in the auxiliary field (vertical and normal to strike) computation (Wannamaker et al., 1985).

The normalized magnetic fields about the conductive circular disk of Figure 2 are given in Figures 6 and 7. These are provided as a check on a 3-D response for other investigators working on thin-layer programs. Note that the normalized field responses of the cisk are greatly diminished compared to that of the 2-D strip even though both are $3 \mathrm{~km}$ across and of $1 . \Omega-m$ resistivity. There are three reasons for this. First, the disk is of short strike extent so that the effective volume of anomalous current is relatively smal1. Second, the profile of field points for the disk is off to the side rather than over as is the case for the 2-D strip. Finally, boundary charges on the disk seriously depress the current density within, and thus the secondary $\mathrm{H}$-fields over, the disk relative to those in a 2-D body of equivalent cross-section (Wannamaker et al., 1984t).

\section{Magnetotelluric Model Study}

The 3-D thin-layer algorithm now is applied to the resistivity geometry studied by Hermance (1982). The geometry is intended to simulate a distributed offset basin of low-resistivity sediments in an otherwise resistive host. The discretization is somewhat different from that of Hermance in order to provide field quantities exactly along profiles to be discussed next.

Specifically, principal apparent resistivities have been calculated at field points along the profiles $A-B$ and $C-D$ considered by Hermance (1982) as well as at field points along extensiors of these profiles outside the basin inhomogeneity. The calculations appear in Figures 8 and 9. In the manner of Hermance, for the purposes of discussian the apparent resistivity of the principal coordinate most nearly parallel to the normal of each profile 


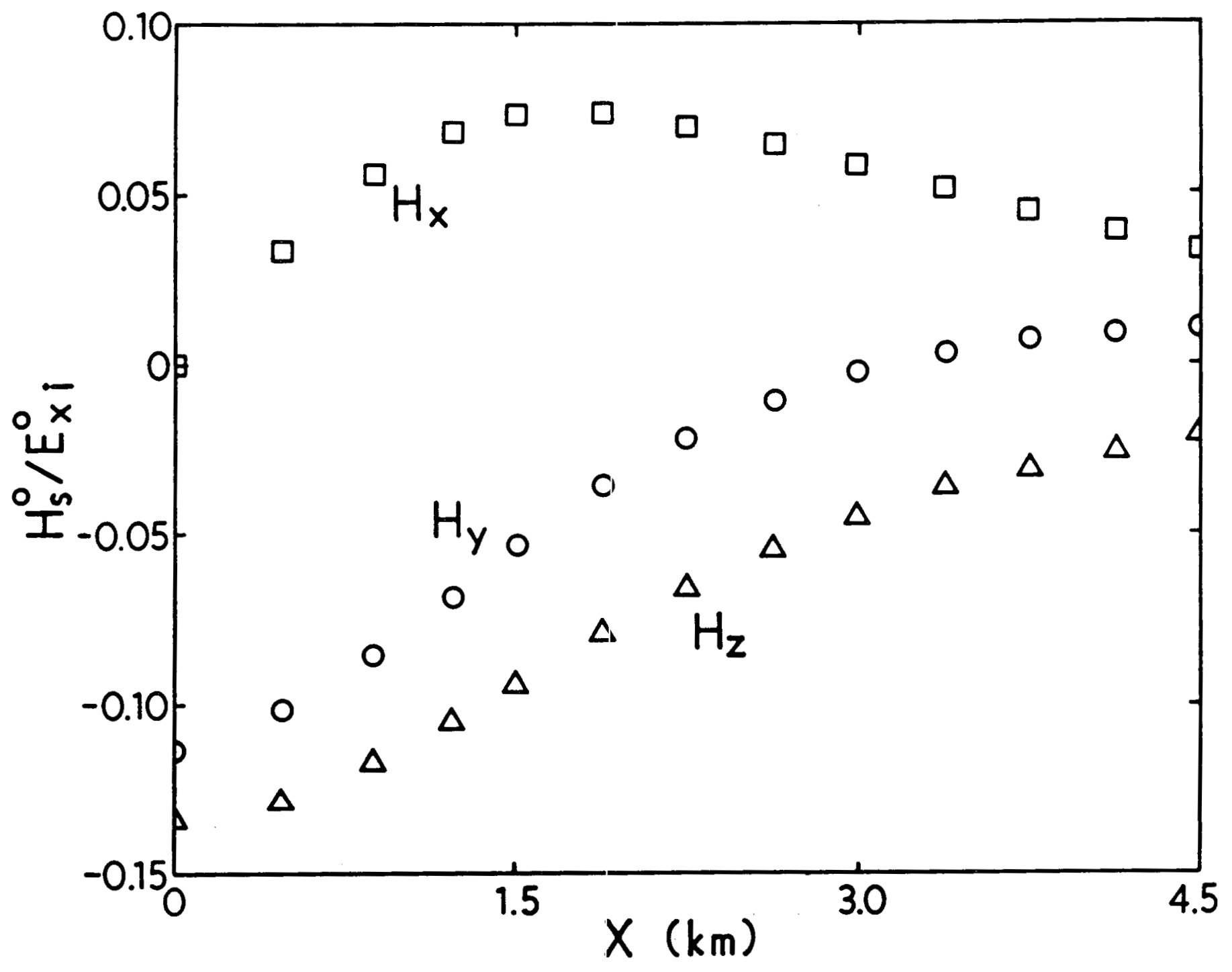

Figure 6. Secondary magnetic field response of conductive disk at field points shown in Figure 2 for an incident electric field along the positive $x$-axis. Field values are normalized by the magnitude of the incident electric field $E_{x i}^{0}$. 


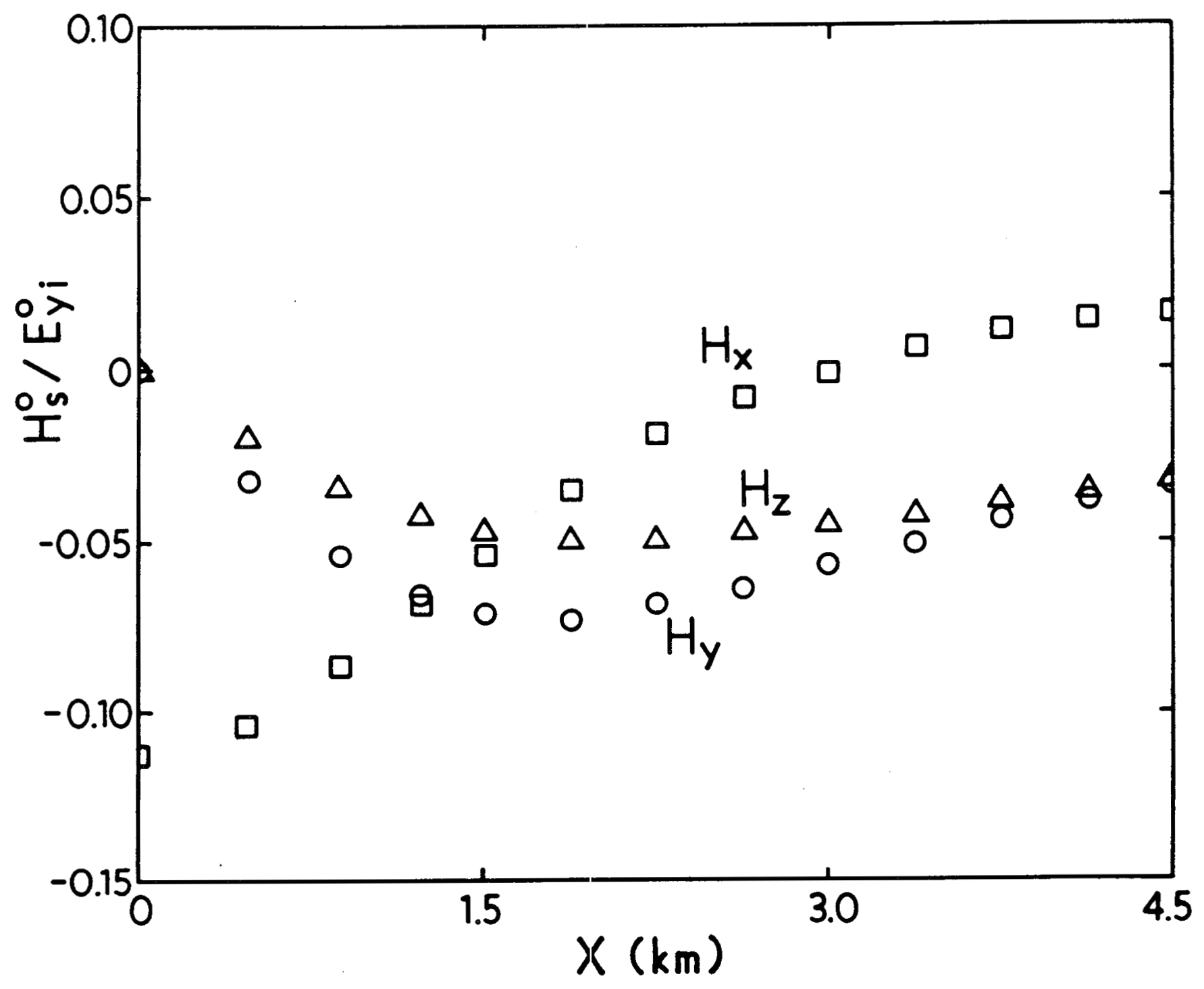

Figure 7. Secondary magnetic field response of conductive disk for an incident electric field along the positive $y$-axis. Field values are normalized by the magnitude of the incident electric field $E_{y i}^{O}$. 


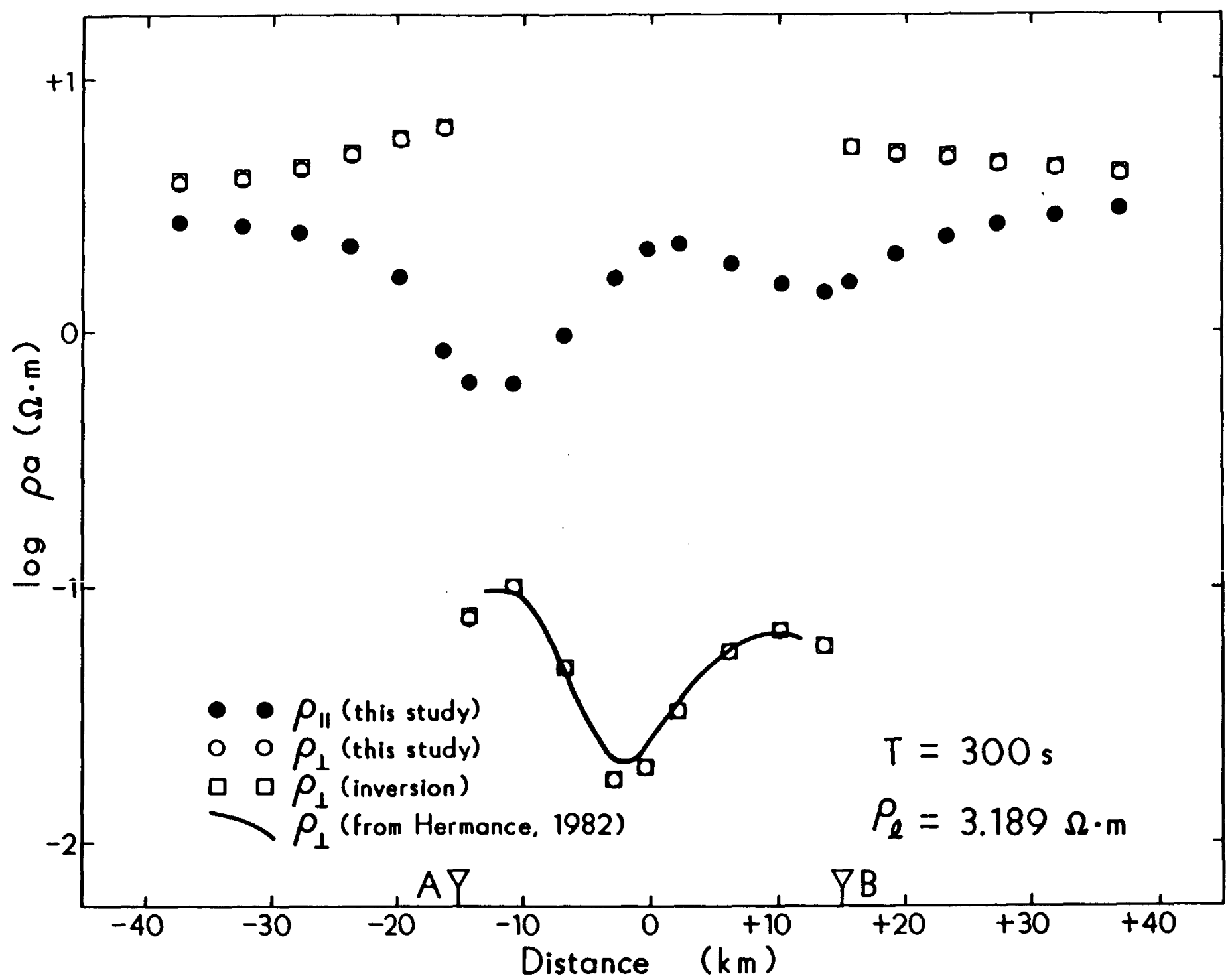

Figure 8. Apparent resistivities of the principal coordinates closest to parallel to the normal to ( $\rho$ ) and perpendicular to the normal to $(\rho$, ) extended profile A-B of MT soundings over the offset basin model of Hermance (1982). Solid curve is the smoothed calculation just within the basin given by Hermance. Finite element discretization in the vicinity of the offset can be inferred from positions of calculated data points on this and the next illustration. 


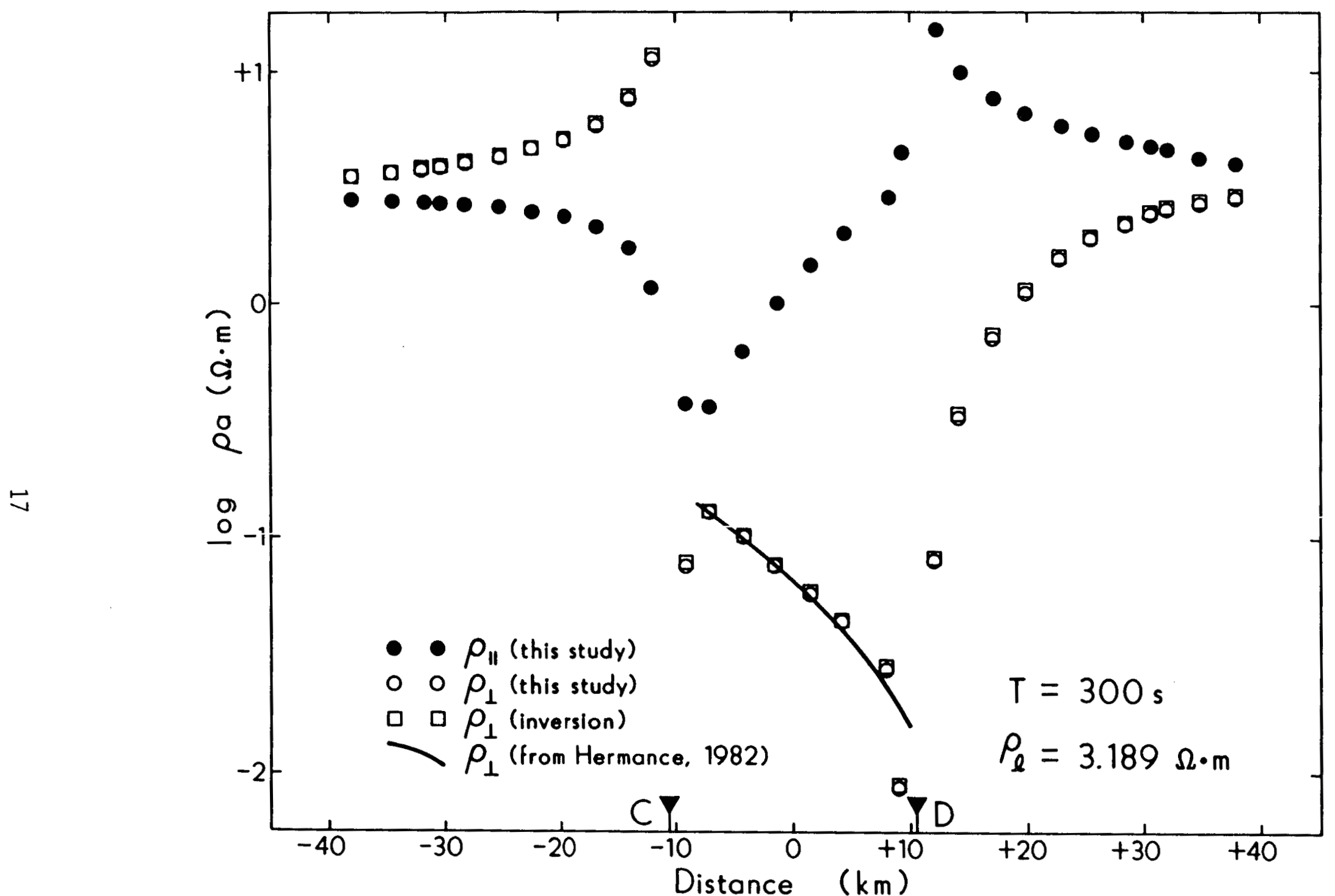

Figure 9. Apparent resistivities of the principal coordinates closest to parallel to the norinal to ( $\rho$ ) and perpendicular to the normal to $\left(\rho_{1}\right)$ extended profile $C-D$ of MT soundings over the offset basin model of Hermance (1982). Solid curve is the smoothed calculation just within the basin given by Hermance. 
$\left(\rho_{\|}\right)$has been identified with the transverse electric (TE) mode while the complementary apparent resistivity $\left(\rho_{\perp}\right)$ has been identified as transverse magnetic (TM).

The agreement between the $\rho_{\perp}$ calculations of Hermance (1982) and of the algorithm described in this report is very good to excellent for the domain of overlap within the low-resistivity basin. Exterior to the basin in Figure 8 , $\rho_{\perp}$ for the extended profile A-B increases discontinously to values of 5 to 6 $\Omega-m$ but decays with further distance to near $3.2 \Omega-m$, which is the apparent resistivity of the layered host used by Hermance at 300 seconds period. The diminishing of $\rho_{\perp}$ outside the basin is a manifestation of the 3-D offset of the basin since transverse magnetic responses are constant with distance outside purely 2-D inhomogeneity for this class of thin-layer structures. For the extended profile $C-D, \rho_{\perp}$ toward the left side of Figure 9 also shows a discontinuous jump from interior values near $0.1 \Omega-m$ to exterior values exceeding $10 \Omega-m$, beyond which $\rho_{\perp}$ decays towards the background value. Toward the right side of Figure $9, \rho_{\perp}$ by contrast shows a discontinuous jump from interior values near $0.01 \Omega-m$ to exterior values around $0.1 \Omega-m$ which increase rapidly with distance toward the background values around $3.2 \Omega-m$. This rather curious behavior outside the conductive basin results from station locations being within a resistive corner and from the oblique orientation of profile C-D with respect to the regional trend of the basin.

Hermance (1982) is correct in noting that the $\rho_{\perp}$ profiles in the vicinity of the 3-D offset differ substantially from the transverse magnetic signatures of purely 2-D structures of cross-sections the same as the resistivity directly beneath the calculated profiles of Figures 8 and 9. The 2-D TM responses should have values around $0.032 \Omega-m$ within the $2-0$ conductor and around $3.2 \Omega-$ 
$m$ outside, with flat behavior as a function of distance otherwise. Certainly, application of a 2-D TM modeling program to data taken across the surficial sedimentary basin as modeled here would lead to errors in estimating the resistivity cross-section of the basin. However, the results of Figures 8 and 9 clearly demonstrate that 2-D transverse electric approaches are no better in such 3-D situations. At the low-frequency limit, $\rho_{\|}$for a purely 2-D basin would register $3.2 \Omega-m$ along the entire length of the profiles. That the calculated values of $\rho_{\|}$I present show strong departures from $3.2 \Omega-m$ comes about due to the presence of electric charge at the resistivity boundaries, charge which is not considered in 2-D TE formulations (Wannamaker et al., 1984b).

Returning to the $\rho_{1}$ computations, Hermance (1982) concluded also that use of a 2-D TM algorithm to model the $\rho_{\perp}$ results across a 3-D surficial basin offset might lead one to interpret false modulations in physical features at depth below the surficial structure. I maintain that such a conclusion cannot be supported on the basis of simulations of this class of thin-layer models. My objection stems from the fact that any profile of apparent resistivity identified as $\rho_{1}$ across 3-D thin-layer inhomogeneity can be fit exactly by an equivalent 2-D thin-layer structure of the same class as considered by Hermance (1982) and in this report.

The equivalent 2-D thin-layer structure may be defined by considering the zero divergence of conduction current at $D C\left(\vec{\nabla} \cdot \vec{J}_{C}=\vec{\nabla} \cdot \sigma \vec{E}=0\right)$. Expanding the divergence-free relation (Harrington, 1961, p. 450), we have

$$
\sigma \bar{\nabla} \cdot \vec{E}=-\vec{E} \cdot \bar{\nabla} \sigma
$$

Letting $y$ be the distance along the profile over the equivalent 2-D body, 


$$
\frac{1}{\sigma} \frac{\partial \sigma}{\partial y}=-\frac{1}{E_{y}} \frac{\partial E_{y}}{\partial y}
$$

or, because the only variation in conductivity or electric field is in the $y$ direction,

$$
\partial \ln \sigma=-\partial \ln E_{y}
$$

Since the apparent resistivity contains the square of the electric field and since I ignore secondary $\mathrm{H}$-fields in this particular study as did Hermance (1982), then

$$
\partial \ln \rho=0.5 \partial \ln \rho_{y x}
$$

where resistivity $p=1 / \sigma$. Integrating from $y=-\infty$, we have

$$
\begin{gathered}
\ln \rho(y) \quad \ln \rho_{y x}(y) \\
\int \partial \ln \rho=0.5 \int \partial \ln \rho_{y x}, \\
\ln \rho(-\infty) \quad \ln \rho_{y x}(-\infty) \\
\ln \rho(y)=\ln \rho(-\infty)+0.5 \ln \left[\rho_{y x}(y) / \rho_{y x}(-\infty)\right]
\end{gathered}
$$

Exponentiating, the inverse giving the equivalent 2-D thin-layer structure is

$$
\rho(y)=\rho(-\infty) \cdot \sqrt{\rho_{y x}(y) / \rho_{y x}(-\infty)}
$$

The simple 2-D inverse of equation (11) has been applied to the calculated $\rho_{\perp}$ profiles along the extended lines $A-B$ and $C-D$ of Figures 8 and 9. The equivalent 2-D thin-layer resistivity variations are plotted in Figure 10 where $\rho(-\infty)$ for the layered host in question is $50 \Omega-m$ and $\rho_{y x}(-\infty)$ is about $3.2 \Omega-m$ (Hermance, 1982). For convenierice of plotting and subsequent test of goodness of fit to the 3-0 calculations, the inverted resistivity is shown in Figure 10 to be constant in the vicinity of a profile data point with boundaries half-way towards adjacent data points. As could be anticipated from 

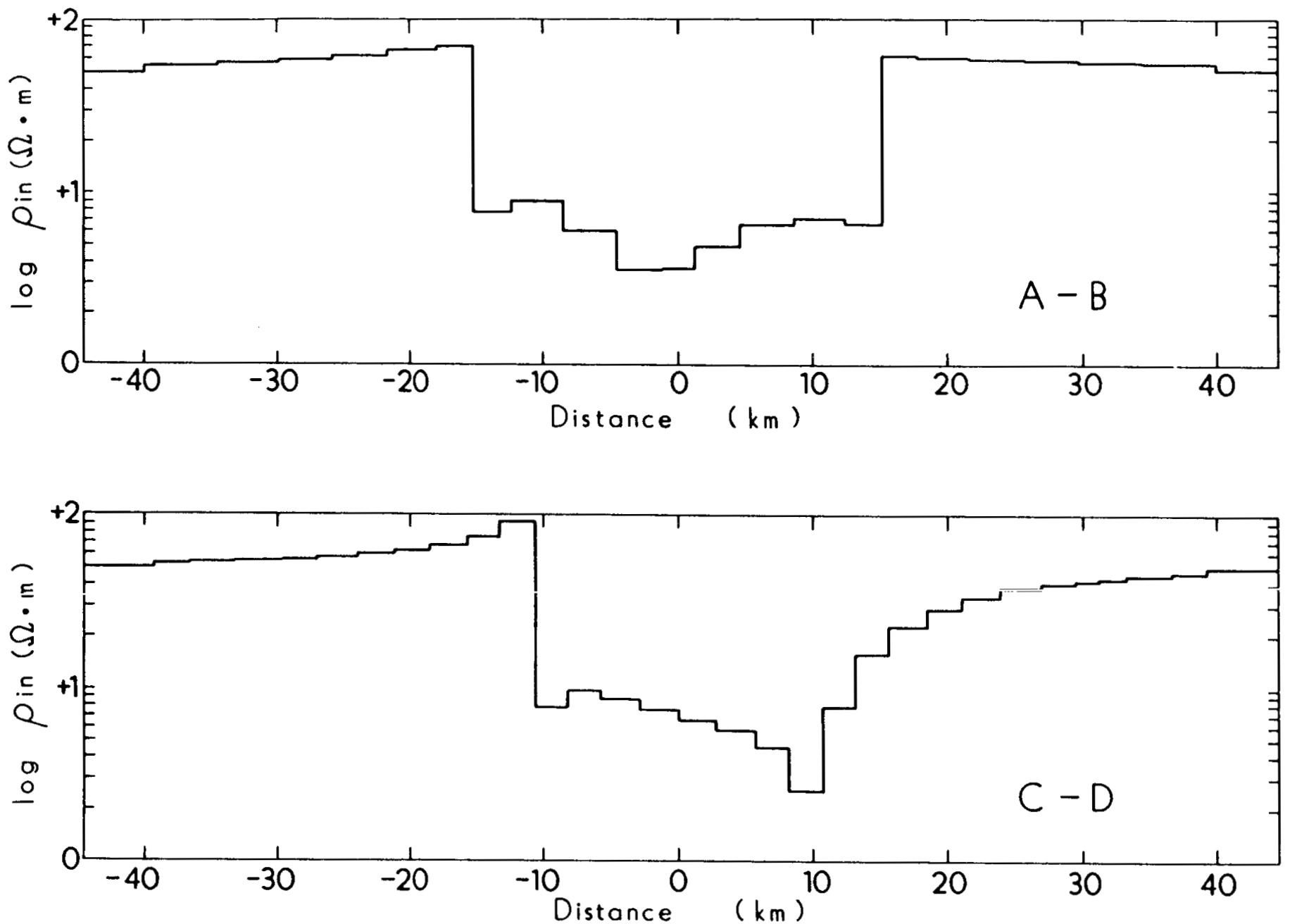

Figure 10. Equivalent 2-D thin-layer resistivity variations obtained by applying equation (11) to calculated $\rho_{\perp}$ values along extended profiles A-B (top) and C-D (hottom) over the 3-D of fset basin model. 
equation (11), the inverted resistivity is a subdued version of the $\rho_{\perp}$ values themselves. Square symbols plotted in Figures 8 and 9 show the excellent fit of the 2-D inverse response, calculated using the thin-layer program, to the original 3-D $\rho_{\perp}$ values. The very slight discrepancies arise from the numerical inaccuracy introduced when the 2-D inverse response is computed using the finite element approximation. Each piecewise constant of anomalous resistivity was represented by only two elements in the simulation.

Equation (11) in fact indicates that an apparent resistivity profile across any 3-D structure may be inverted precisely to yield an equivalent 2-D thin-layer variation of the same class as considered herein because the apparent resistivity is a real quantity never less than zero. Since such an equivalence is possible, then conjecture that errors in the interpretation of resistivity at depth below surficial inhomogeneity occur when a 2-D TM algorithm is applied to profiles of soundings across 3-0 structure cannot be supported merely by 3-D thin-layer simulations of the type employed by Hermance (1982) or in this report. One could as readily conclude from just the thin-layer investigations that 2-D TM modeling across a 3-D body at the surface yields an equivalent 2-D cross-section residing in the same regional structure (the layered host in this study) as the 3-D body. A11 that may be demonstrated firmly by these thin-layer simulations is that errors in estimates of the surficial structure could result from a 2-D TM application. Note also that the inverse of (11) does not depend on the definition of $\rho_{\perp}\left(=\rho_{y x}(y)\right.$ in inverse) used, so that an exact simulation will result whether principal axis or fixed coordinate (Wannamaker et al., 1984b) data are chosen. 


\section{DIPOLE-DIPOLE RESPONSES}

\section{Checks on the Calculated Potentials}

To test the accuracy of the thin layer algorithm for computing electrostatic potentials due to current sources, I return to the conductive disk model of Figure 2. Apparent resistivities of a pole-bipole array according to equation (3) are obtained from calculated voltages across pairs of nodes as shown with the $x^{\prime} s$ in Figure 2. The profile also is extended symmetrically in the negative $x$ direction to give additional apparent resistivities. Defining the radius of the disk as one unit, the apparent resistivity profile is located along the line $y=-2$ and three single sources in turn are considered along the line $x=-2$ at positions $y=-1,0$ and +1 . The resistivity contrast between the disk and the host is 0.538 .

The apparent resistivities computed by the thin-layer program for the three pole-bipole arrays are presented in Figures 11, 12 and 13 and have been normalized by the host resistivity $\rho_{1}$. The apparent resistivity data of these figures are plotted at the midpoints of the nodal locations of Figure 2 . The thin-layer results are compared to surface integral equations calculations for a conductive cylinder adjacent to a 2-D continuously-grounded current source. The surface integral equations program is decribed by Yang and Ward (1985) and is modified from the method of Barnett (1972).

The comparison between the two methods of calculation is good overall with the worst agreement occurring for points between the body and the source, especially the deepest source. It is unclear which solution is better. I point out that either method shows an apparent resistivity discontinuity as the receiver bipole passes over the source, a discontinuity which seems especially puzzling for the surface integral technique since the secondary 


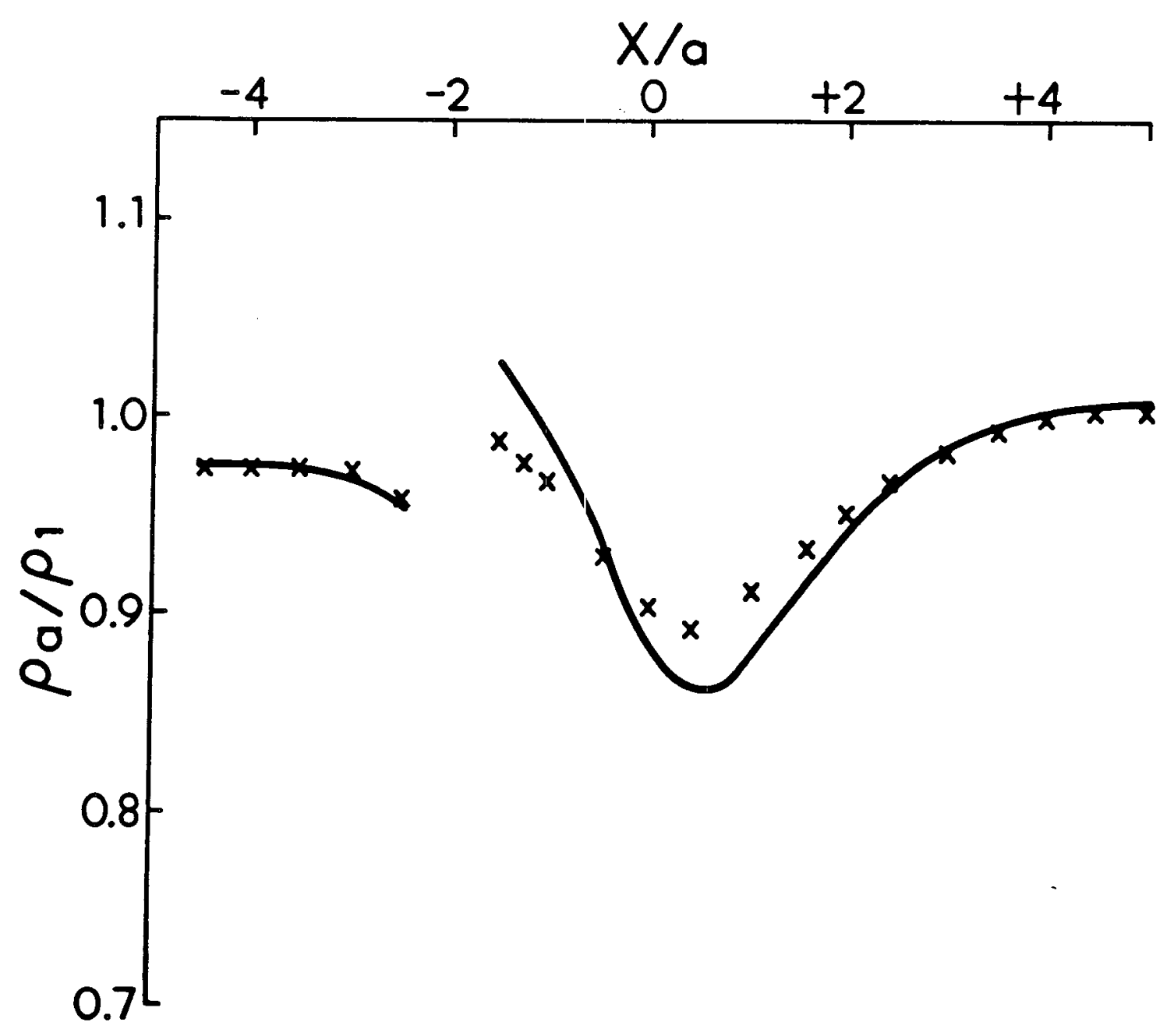

Figure 11. Normalized apparent resistivities for pole-bipole array calculated using finite elements ( $x^{\prime} s$ ) and using surface integral equations (solid curve) for the pole source at $y=+1$ units and $x=-2$ units outside the conductive disk model of Figure 2. The apparent resistivity data of this and the next two figures are plotted at the midpoints of the nodal locations of Figure 2. The radius of the disk by which dimensions are normalized is a. 


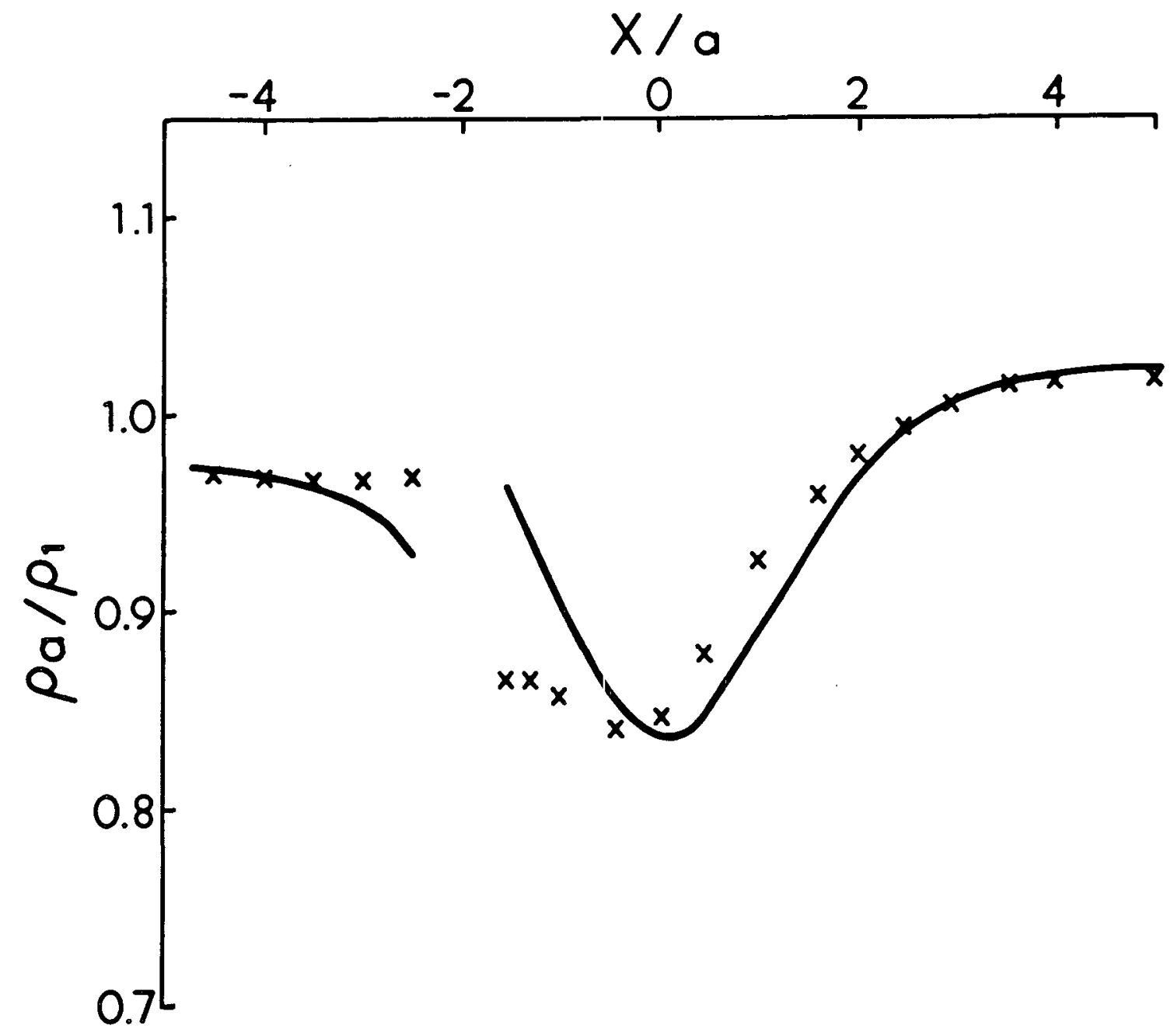

Figure 12. Normalized apparent resistivities for pole-bipole array calculated using finite elements ( $x^{\prime} s$ ) and using surface integral equations (solid curve) for the pole source at $y=0$ units and $x=-2$ units outside the conductive disk model of Figure 2. 


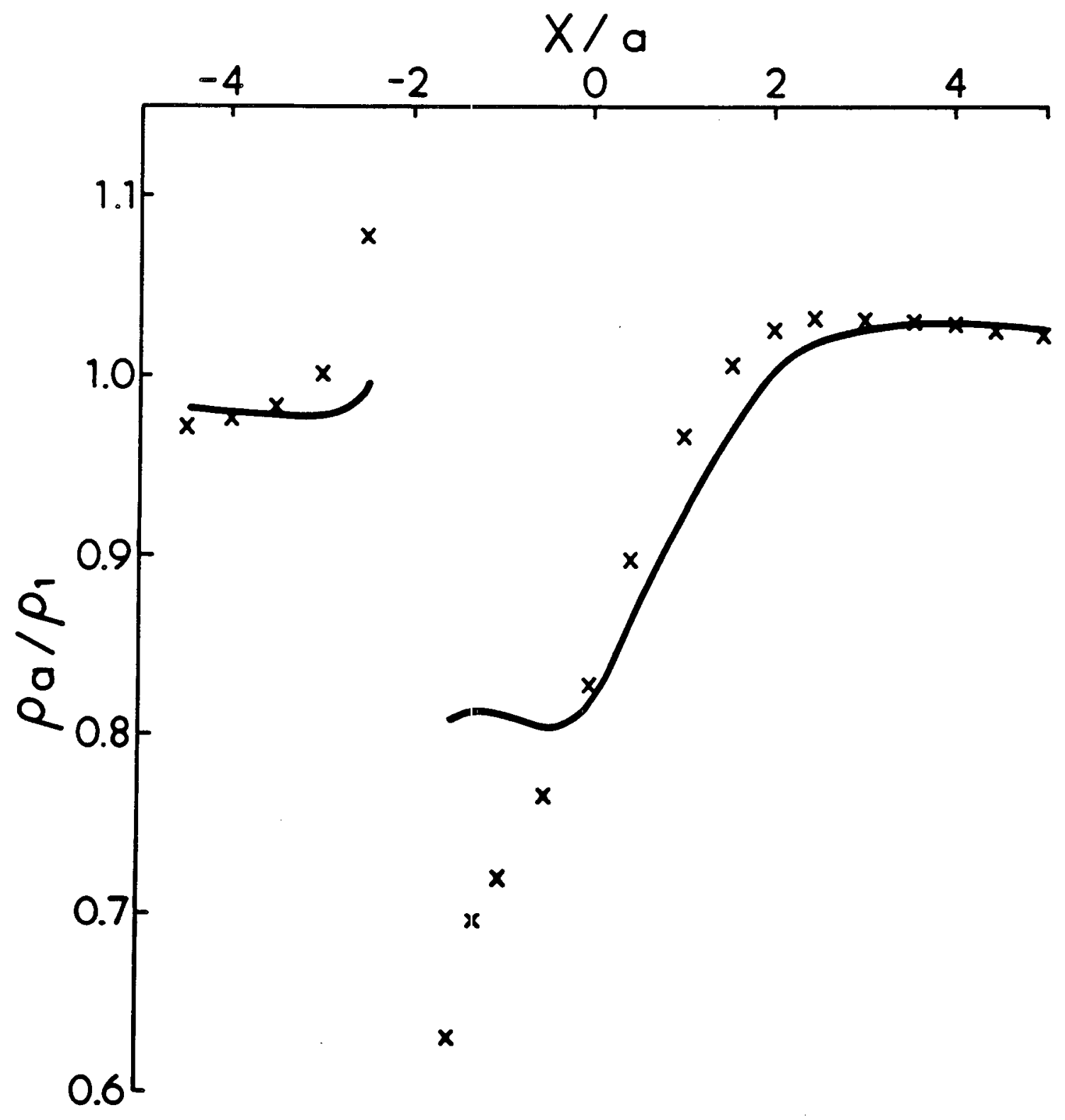

Figure 13. Normalized apparent resistivities for pole-bipole array calculated using finite elements ( $\left.x^{\prime} s\right)$ and using surface integral equations (solid curve) for the pole source at $y=-1$ units and $x=-2$ units outside the conductive disk model of Figure 2. 
potentials result from integration of boundary charge just over the surface of the body. Obtaining good results above the source is difficult because the potential differencing selected cuts across the primary current flow. The potential differences are smal1, and are normalized by small geometric factors, so that small absolute errors would result in large percentage errors in apparent resistivity.

\section{Dipole-Dipole Model Study}

For a model study with the dipole-dipole array, I simply consider the example thin-layer structure of Figure 1. The model is meant to portray a connected pair of sedimentary distributions in an otherwise resistive host.

The dipole-dipole response of this body appears in Figure 14, first for the structure of the same resistivity as the host $(100 \Omega-m)$ and then for the structure of $5 . \Omega-m$. The first instance tests the ability of the finite element method to simulate the primary potential distribution and tests the correctness of the geometric factors for the dipole-dipole array. The calculations appear to be within one percent of the true value of $100 \Omega$-m over the majority of the pseudosection.

When the alluvium is given a low resistivity, strong apparent resistivity anomalies appear in the pseudosection of figure 14. The effect of conductive material trending obliquely to the side of the dipole-dipole array is to suggest a conductor dipping under the array. Apparent resistivities less than 5. $\Omega-m$ are observed. The calculations suggest that surficial alluvium must be accounted for in dipole-dipole surveys as well as magnetotelluric surveys in order for deeper resistivity structure to be resolved. Petrick et al. (1981) experienced the overwhelming effects of similar near-surface sediments in their dipole-dipole measurements near the Roosevelt Hot Springs thermal area. 
APPARENT RESISTIVITY

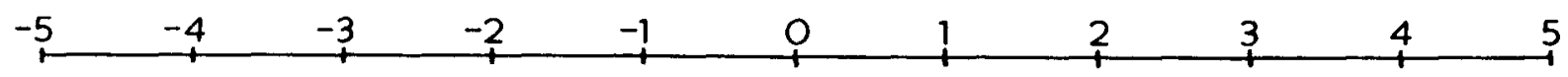

\begin{tabular}{|c|c|c|c|c|c|c|c|c|c|c|c|c|c|c|c|c|c|c|}
\hline & & 99 & & 99 & & 100 & & 100 & & 99 & & 99 & & 99 & & 99 & & \\
\hline & 100 & & 100 & & 100 & & 100 & & 100 & & 100 & & 100 & & 100 & & 100 & \\
\hline 100 & & 100 & & 100 & & 100 & & 100 & & 100 & & 100 & & 100 & & 100 & & 100 \\
\hline וכ & 101 & & 100 & & 100 & & 100 & & 100 & & 100 & & 100 & & 100 & & 101 & 101 \\
\hline 101 & & 101 & & 101 & & 101 & & 101 & & 101 & & 101 & & 101 & & 101 & & 101 \\
\hline & 101 & & 101 & & 101 & & 101 & & & & 101 & & 101 & & 101 & & 101 & \\
\hline
\end{tabular}
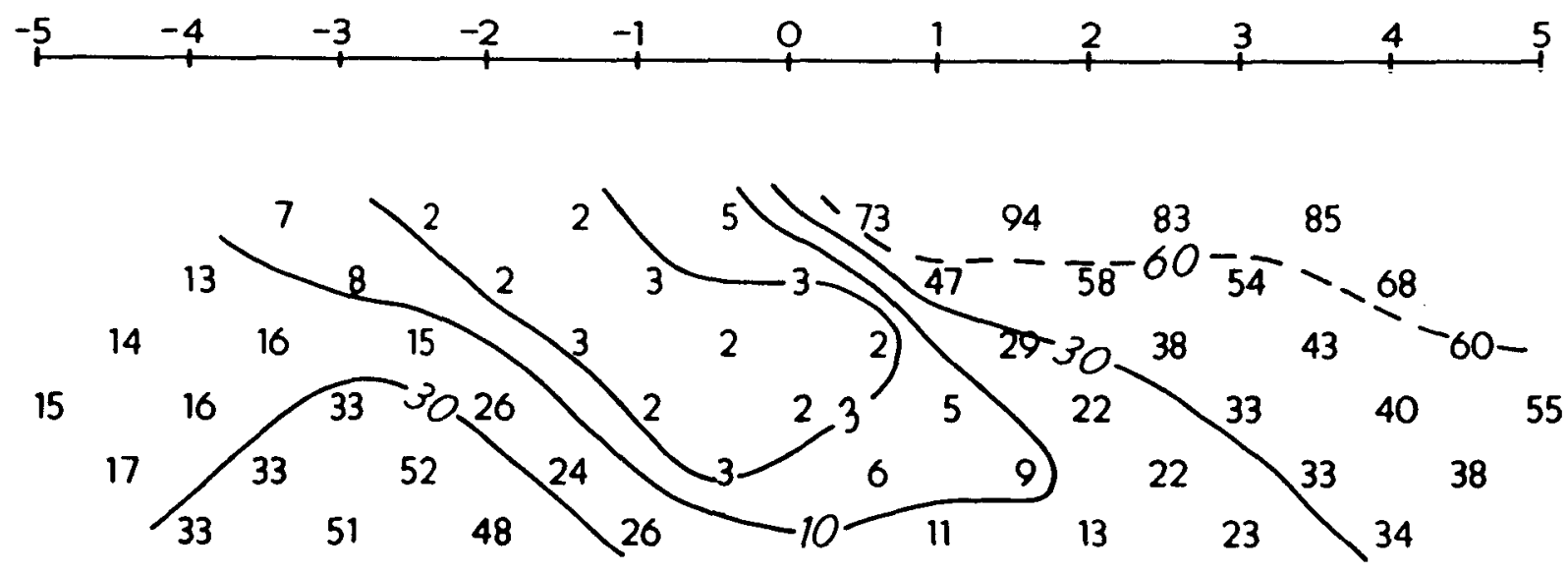

Figure 14. Dipole-dipole apparent resistivities computed by the thin-layer program for the example thinlayer structure of Figure 1 . At the top the results with $\rho_{2}=\rho_{1}=100 \Omega-m$ test the ability of the finite elements to reproduce the primary voltage distribution and apply the geometric factors correctly. At the bottom is the response when the 3-0 structure is given a resistivity $\rho_{2}=5$. $\Omega-m_{\text {. }}$ 
By reducing the degree of complexity from three-dimensional to essentially two-dimensional, 3-0 thin-layer structure of the class considered in this report can be simulated with relative ease on the computer. Lowfrequency limits to responses are useful to know as they often represent the strongest anomalies for 3-D bodies. Imposition of the infinitely resistive basement also tends to maximize the areal extent of an anomaly by preventing return current flow through the basement (see Wannamaker et al., 1984b). Integration of secondary current perturbations over the thin sheet is an accurate method of obtaining anomalous magnetic fields, but it constitutes the most time-consuming part of the problem for more than about a dozen receiver points even though simple analytic free-space Green's functions are used.

It is hazardous however to apply such a thin-layer program beyond simple order-of-magnitude forward calculations. For example in MT, supposition that 2-D TM mode interpretation approaches to defining deep structure below surficial heterogeneity may suffer pitfalls due to the 3-D nature of the surficial structure cannot be upheld just on the basis of very low frequency, 3-D thin-layer calculations. This is because any single-frequency profile of apparent resistivities assigned to the TM mode across a 3-D structure may be fit precisely by an equivalent 2-D structure whose entire resistivity variation is limited to an outcropping thin-layer. Worthwhile future research would pursue definition of an equivalent 3-D thin-layer structure which could simulate single-frequency areal distributions of MT apparent resistivities over arbitrary 3-D resistivity at any frequency. 


\section{ACKNOWLEDGEMENTS}

This report was funded by U. S. Department of Energy Contract No. DEAC03-84SF12196. 
Barnett, C. T., 1972, Theoretical modeling of induced polarization effects due to arbitrarily shaped bodies: Ph.l). thesis, Colorado School of Mines.

Dawson, T. W., and Weaver, J. T., 1979, Three-dimensional induction in a nonuniform thin sheet at the surface of a uniformly conducting earth: Geop. J. Roy. Astr. Soc., 59, 445-462.

Fox, R. C., Hohmann, G. W., Killpack, T. J., and Rijo, L., 1980, Topographic effects in resistivity and induced polarization surveys.

Harrington, R. F., 1961, Time-harmonic electromagnetic fields: McGraw-Hill Electrical and Electronic Engineering Series, MCGraw-Hill Book Co., Inc., New York, $480 \mathrm{p}$.

Hermance, J. F., 1982, The asymptotic response of three-dimensional basin offsets to magnetotelluric fields at long periods: the effects of current-channeling: Geophysics, 4\%, 1562-1573.

Huebner, K. H., and Thornton, E. A., 1982, The finite element method for engineers: John Wiley \& Sons, New York, 623 p.

Kellogg, 0. D., 1953, Foundations of potential theory: Dover Publications, Inc., New York, $384 \mathrm{p}$.

McKirdy, D. MCA., weaver, J. T., and Dälwson, T. W., 1985, Induction in a thin sheet of variable conductance at the surface of a stratified earth-II. Three-dimensional theory: Geop. J. Roy. Astr. Soc., 80, 177-194.

Park, S. K., Orange, A. S., and Madden, T. R., 1983, Effects of threedimensional structure on magnetotelluric sounding curves: Geophysics, 48, 1402-1405.

Petrick, W. R., sill, W. R., and Ward, S. H., 1981, Three-dimensional resistivity inversion using alpha centers: Geophysics, 46, 1148-1162.

Ranganayaki, R. P., and Madden, T. R., 1980, Generalized thin sheet analysis in magnetoteliurics: an extension of Price's analysis: Geop. J. Roy. Astr. Soc., 60, 445-457.

Rijo, L., 1977, Modeling of electric and electromagnetic data: Ph.D. thesis, Univ. of Utah, $242 \mathrm{p}$.

Vasseur, G., and Weidelt, P., 1977, Bimodal electromagnetic induction in nonuniform thin sheets with an application to the northern Pyrenean induction anomaly: Geop. J. Roy. Astr. Soc., 51, 669-690.

Wannamaker, P. F., Hohmann, G. W., and SanFilipo, W. A., 1984a, Electromagnetic modeling of three-dimensional bodies in layered earths using integral equations: Geophysics, 49, 60-74. 
Wannamaker, P. E., Hohmann, G. W., and Ward, S. H., 1984b, Magnetotelluric

- responses of three-dimensional bodies in layered earths: Geophysics, 49, $1517-1533$.

Wannamaker, P. E., Stodt, J. A., and Rijo, L., 1985, PW20 - finite element - program for solution of magnetotelluric responses of two-dimensional earth resistivity structure: Univ. of Ut:ah Research Inst. Report ESL-158, 71 p.

Weidelt, P., 1971, The electromagnetic induction in two thin half-sheets: $Z$. Geophysik, 37, 649-665.

Yang, F. W., and Ward, S. H., 1985, Single- and cross-borehole resistivity anomalies of thin ellipsoids and shells: Geophysics, 50, 637-655. 